\title{
Different Attitudes of Patients and Psychiatrists Toward Benzodiazepine Treatment
}

\author{
Yasushi Kawamata' \\ Norio Sugawara $\mathbb{D D}^{1,2}$ \\ Masamichi Ishioka $\mathbb{D}^{3}$ \\ Kazutoshi Kubo ${ }^{4}$ \\ Katsuji Suzuki ${ }^{5}$ \\ Akira Fujii ${ }^{6}$ \\ Hanako Furukori ${ }^{7}$ \\ Taku Nakagami ${ }^{8}$ \\ Norio Yasui-Furukori (ID) ${ }^{1,2}$ \\ Kazutaka Shimoda' \\ 'Department of Psychiatry, Dokkyo \\ Medical University School of Medicine, \\ Mibu, Japan; ${ }^{2}$ Department of \\ Neuropsychiatry, Hirosaki University \\ Graduate School of Medicine, Hirosaki, \\ Japan; ${ }^{3}$ Department of Psychiatry, Minato \\ Hospital, Hachinohe, Japan; ${ }^{4}$ Department \\ of Neuropsychiatry, Hirosaki-Aiseikai \\ Hospital, Hirosaki, Japan; ${ }^{5}$ Department of \\ Psychiatry, Aomori Central Hospital, \\ Aomori, Japan; ${ }^{6}$ Department of \\ Neuropsychiatry, Mutsu General \\ Hospital, Mutsu, Japan; ${ }^{7}$ Department of \\ Neuropsychiatry, Kuroishi-Akebono \\ Hospital, Kuroishi, Japan; ${ }^{8}$ Department of \\ Psychiatry, Nakagami Mental Clinic, \\ Ohdate, Japan
}

Background: Concern regarding the benefit/risk ratio of the long-term use of benzodiazepines (BDZs) and Z-drugs is increasing. To prevent the risk of dependence in BDZ long-term use, it is essential to understand the attitudes of patients and psychiatrists toward BDZ treatment. The aims of this investigation were to 1) obtain information on patients' attitudes with long-term BDZ use and their referring psychiatrists' attitudes toward BDZ treatment, including their perception of the difficulty of reducing the dose of BDZs, and 2) identify discrepancies between patients' and psychiatrists' perceptions.

Methods: A brief questionnaire was constructed to investigate the attitudes of patients receiving BDZ treatment and their referring psychiatrists. Our sample comprised 155 patients who received BDZ treatment for more than one year and their referring eight psychiatrists. Both the patients and their psychiatrists completed our questionnaire between August 2017 and December 2017.

Results: Of the patients, $13 \%$ felt that it was more difficult to reduce the dose of BDZs than their referring psychiatrists (type A discrepancy), while $25 \%$ felt that it was less difficult (type B discrepancy). In the multivariate logistic regression analysis, the female sex and both the patients' ("psychotherapy plus BDZs was necessary" and "it was necessary to increase the dose of BDZs") and psychiatrists' beliefs ("short-term prescription was justified") were associated with type A discrepancies. Type B discrepancies were associated with psychiatrists' beliefs that the patient's wishes justified the use of BDZs and that the cessation of treatment with BDZs would lead to the deterioration of their rapport with their patients.

Conclusion: To overcome the discrepancies in the attitudes of patients and psychiatrists toward the cessation of BDZ treatment, it is necessary to promote patient-centered care involving patient psychoeducation and practice guidelines for the decision-making process. Further studies investigating the promotion of patient-centered care to reduce BDZ use are needed.

Keywords: dependence, benzodiazepine receptor agonist, anxiolytics, hypnotics, belief

\section{Introduction}

Concern regarding the benefit/risk ratio of the long-term use of benzodiazepines (BDZs) and Z-drugs is increasing. While BDZs have several therapeutic effects, including anxiolytic, hypnotic, muscle relaxing, and anticonvulsant actions, the adverse effects (drowsiness, fatigue, disturbance of concentration and attention, and development of dependence) of BDZs are problematic. Although the longterm use of BDZs is not recommended, ${ }^{1}$ a high prevalence of long-term treatment with BDZs has been reported. ${ }^{2,3}$ To prevent the risk of dependence in BDZ longterm use, it is essential to understand the attitudes of patients and psychiatrists toward BDZ treatment.
Correspondence: Norio Yasui-Furukori Department of Psychiatry, Dokkyo Medical University School of Medicine, 880 Kitakobayashi, Mibu, Tochigi, 32I-0293, Japan

Tel $+8 \mathrm{I}-28-287-2153$

Fax +8I-28-286-5187

Email furukori@dokkyomed.ac.jp 
There have been several reports regarding patients' attitudes toward BDZ treatment. In the Swedish primary health care setting, Magnúsdóttir and Ribacke investigated the knowledge and attitudes regarding hypnotics among patients treated by physicians. They showed that most patients were well informed about the benefits and possible addictive effects. ${ }^{4}$ With regard to the motivations to discontinue treatment with this medication, a qualitative study conducted in Switzerland indicated that high-dose BDZ-dependent patients' decisions to change their consumption patterns were affected by health concerns, the feeling of being addicted and social factors. ${ }^{5}$ Another qualitative study from Australia investigated attitudes toward the cessation of long-term nocturnal BDZ treatment among patients older than 65 years. ${ }^{6}$ They found that many patients expressed a willingness to cease nocturnal BDZ use, while general practitioners (GPs) continued to prescribe the medications.

Anthierens and colleagues studied physicians' attitudes toward BDZ treatment and perceived barriers to nonpharmacologic approaches to treating psychological distress. Of the GPs, $47.2 \%$ thought that patients would expect a prescription, $17.8 \%$ were afraid that not writing a prescription would damage the GP-patient relationship, and $60.6 \%$ felt that convincing patients to stop taking BDZs would be difficult. ${ }^{7}$ Based on previous studies investigating the attitudes of patients or psychiatrists, we hypothesized that the attitudes toward BDZ of patients and psychiatrists differ substantially.

To our knowledge, no studies have addressed the abovementioned differences in attitudes. The objectives of this study were to 1) obtain information on patients' attitudes toward long-term BDZ use and their referring psychiatrists' attitudes toward BDZ treatment, including their perception of the difficulty of dose reduction and 2) identify discrepancies between patients' and psychiatrists' attitudes toward BDZ dose reductions.

\section{Methods}

\section{Participants}

This study was conducted between August 2017 and December 2017. The data collection protocol for this study (2017-1049) was approved by the Ethics Committee of the Hirosaki University School of Medicine, and all subjects provided written informed consent before participating in this study. This protocol was conducted in accordance with the principles of the
Declaration of Helsinki and the Japanese Ethical Guidelines for Medical and Health Research Involving Human Subjects.

The subjects included 155 outpatients (54 males and 101 females) who were on long-term (one year or more) BDZ treatment and their referring eight psychiatrists at seven psychiatric hospitals in Japan. Participants were selected based on the available sampling method. The diagnoses of the patients according to the Diagnostic and Statistical Manual of Mental Disorders, fifth edition, or the International Classification of Disease, tenth revision, were obtained from their medical charts.

The medical records of the patients were reviewed to obtain data on diagnoses, duration of illness, duration of BDZ use, medications, and demographic data (age, sex), and the Clinical Global Impression-Severity scale (CGI-S) was assessed. Participants completed questionnaires that asked about their lifestyle factors (smoking status, habitual alcohol intake, frequency of driving a car); frequency of $\mathrm{BDZ}$ use; previous BDZ cessation attempts as indicated by psychiatrists; and adherence. Regarding habitual alcohol intake, participants were asked about their frequency of alcohol consumption. Those answering "not at all" were considered the "no habitual alcohol intake" group, while those answering "sometimes" or "everyday" were considered the "habitual alcohol intake" group. Regarding BDZs, we converted each BDZ to diazepam (DZP) equivalents for the purposes of comparison. ${ }^{8}$ According to the package insert, each BDZ, with the exclusion of Z-drugs, was classified into one of three groups (short half-life group, intermediate half-life group, long half-life group).

After reviewing previous investigations regarding the attitudes of patients or psychiatrists, ${ }^{4-7}$ we developed an original questionnaire (Q1-Q18) concerning attitudes toward BDZ use. The questions directed towards patients were answered on a 4-point scale (1=strongly disagree, $2=$ disagree, $3=$ agree, $4=$ strongly agree). The questions direct towards psychiatrists were answered on a 5-point scale $(0=$ not applicable, $1=$ strongly disagree, $2=$ disagree, $3=$ agree, $4=$ strongly agree). Those answering 1 or 2 were grouped in the "disagree" group, and those responding with 3 or 4 were classified in the "agree" group. In the logistic regression analysis described below, "not applicable" and "disagree" were grouped as "others".

Concerning difficulties with BDZ dose reduction, a type A discrepancy was identified when the patient perceived reducing the dose to be difficult when the referring psychiatrist did not, while a type B discrepancy was identified when 
the patient did not perceive reducing the dose to be difficult when the referring psychiatrist did (Figure 1).

\section{Statistical Analysis}

The data are presented as the mean and standard deviation (SD) and number (\%). The perception of the difficulty of reducing the dose of BDZ was analyzed with stratification by age and sex. Chi-square tests were performed for categorical variables. A value of $p<0.05$ was considered significant. Multivariate logistic regression analysis with a forward selection method was performed to assess the effects of the patients' attitudes (Q2-Q18 for patients) and covariates (age, sex, occasional use of BDZs, previous BDZ cessation attempts indicated by psychiatrist, duration of illness, duration of BDZ use, CGI-S, frequency of smoking, frequency of alcohol consumption, frequency of driving cars, adherence, diagnosis, DZP equivalent dose of the total prescription, use of short half-life BDZs except for Z-drugs, use of intermediate half-life BDZs except for Z-drugs, use of long half-life BDZs except for $Z$-drugs and use of Z-drugs) on the perception of difficulty in reducing the dose of BDZs (an "agree" response to Q1 by the patients was scaled as 1 , and the response "others" was scaled as 0 ). The effects of the psychiatrists' attitudes

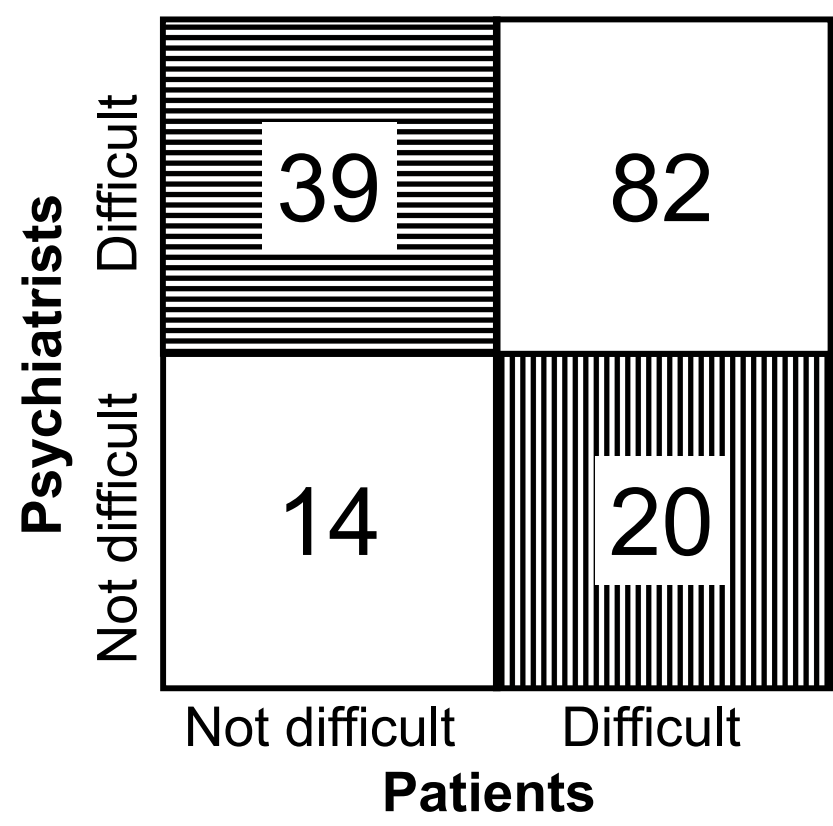

Figure I Responses of patients and their referring psychiatrists regarding the difficulty of reducing the dose of BDZs.

Notes: The box shaded with vertical lines shows a type A discrepancy (patients perceive a reduction in the dose of BDZs to be more difficult than their referring psychiatrists do). The box shaded with horizontal lines shows a type B discrepancy (patients perceive a reduction in the dose of BDZs to be less difficult than their referring psychiatrists do).
(Q2-Q18 for the psychiatrists) and the same covariates on the perception of difficulty with regard to reducing the dose of BDZs (an "agree" response to Q1 by the psychiatrists was scaled as 1 , and the response "others" was scaled as 0 ) were also assessed. Furthermore, we also conducted multivariate logistic regression analysis to assess the effects of the patients' attitudes (Q2-Q18 for patients), the psychiatrists' attitudes (Q2-Q18 for psychiatrists) and the covariates on type A and B discrepancies. Regarding the dependent variables, the type A discrepancy group was scaled as 1, the other group was scaled as 0, the type B discrepancy group was scaled as 1, and the other group was scaled as 0 . The data were analyzed using SPSS software for Windows (Version 26.0.0.0).

\section{Results}

The clinical and demographic characteristics of the participants are listed in Table 1. Table 2 shows the patients' attitudes toward BDZs, and Table 3 shows the psychiatrists' attitudes toward BDZs. The question with the most common agreement between patients and psychiatrists was Q9: "Do you believe that the continued administration of BDZs should continue as long as you desire?" (89.0\%) and Q13 "Do you believe that information about addiction is important?" (98.1\%). Table 4 shows the perception of difficulty in reducing the $\mathrm{BDZ}$ dose (Q1) as evaluated by the patients and their referring psychiatrists. There were no significant differences in age or sex among the categories.

Table 5 shows the factors associated with the patients' and psychiatrists' perception of a dose reduction being difficult (Q1). To assess the influence of attitudes (Q2Q18) or characteristics on perception of difficulty (Q1), we performed a multivariate logistic regression analysis with a forward selection method. DZP equivalent doses of short half-life BDZs except for Z-drugs and patients' responses to Q6, Q16 and Q17 were significantly associated with patients perceiving a dose reduction as being difficult (Q1 for the patients). The duration of BDZ use, frequency of driving cars, frequency of alcohol consumption, and psychiatrists' responses to Q2, Q5, Q9, Q16, Q17 and Q18 were significantly associated with psychiatrists' perception of a dose reduction as being difficult (Q1 for the psychiatrists). Table 6 shows the factors associated with discrepancies in attitudes between patients and psychiatrists regarding the difficulty of BDZ dose reduction. To assess the influence of attitudes (Q2-Q18 for patients or psychiatrists) and characteristics on these discrepancies (type A or type B), we performed a multivariate logistic 
Table I Clinical and Demographic Characteristics. Values are the Mean and Standard Deviation (SD) or Number (\%)

\begin{tabular}{|c|c|}
\hline Characteristics & $\begin{array}{r}\text { All } \\
(N=155)\end{array}$ \\
\hline Age (years), mean (SD) & $48.2(15.9)$ \\
\hline Female sex, $\mathrm{n}(\%)$ & $101(65.2 \%)$ \\
\hline Occasional (not daily) use of BDZs, n (\%) & $8(5.2 \%)$ \\
\hline $\begin{array}{l}\text { Previous BDZ cessation attempts indicated by } \\
\text { psychiatrist, } \mathrm{n}(\%)\end{array}$ & $16(9.3 \%)$ \\
\hline Duration of illness (years), mean (SD) & $11.7(7.6)$ \\
\hline Duration of BDZ use (years), mean (SD) & II (7.I) \\
\hline CGI-S, mean (SD) & $3.2(1.2)$ \\
\hline Smoking, n (\%) & \\
\hline Not at all & $108(69.7 \%)$ \\
\hline Sometimes & $2(1.3 \%)$ \\
\hline Everyday & $45(29.0 \%)$ \\
\hline Alcohol consumption, n (\%) & \\
\hline Not at all & $100(64.5 \%)$ \\
\hline Sometimes & $45(29.0 \%)$ \\
\hline Everyday & $10(6.5 \%)$ \\
\hline Driving cars, n (\%) & \\
\hline Not at all & 69 (44.5\%) \\
\hline Sometimes & $29(18.7 \%)$ \\
\hline Everyday & $57(36.8 \%)$ \\
\hline Adherence, $\mathrm{n}(\%)$ & \\
\hline Taking BDZs as prescription & $135(87.1 \%)$ \\
\hline Forgetting to take BDZs sometimes & 7 (4.5\%) \\
\hline Adjust BDZs by myself & $13(8.4 \%)$ \\
\hline Diagnosis, n (\%) & \\
\hline Schizophrenia spectrum & $44(27.7 \%)$ \\
\hline Bipolar disorder & $13(8.4 \%)$ \\
\hline Major depressive disorder & $40(25.8 \%)$ \\
\hline Anxiety disorders & $43(27.7 \%)$ \\
\hline Autism spectrum disorder & $6(3.9 \%)$ \\
\hline Others & $9(5.8 \%)$ \\
\hline Total DZP equivalent dose $(\mathrm{mg})$, mean (SD) & $14.3(11.9)$ \\
\hline $\begin{array}{l}\text { DZP equivalent dose of BDZs except for z-drugs } \\
(\mathrm{mg}) \text {, mean (SD) }\end{array}$ & |3.4 ( 1 I.8) \\
\hline $\begin{array}{l}\text { Short half-life BDZs except for z-drugs (mg), } \\
\text { mean (SD) }\end{array}$ & $3.4(4.8)$ \\
\hline $\begin{array}{l}\text { Intermediate half-life BDZs except for z-drugs } \\
(\mathrm{mg}) \text {, mean }(\mathrm{SD})\end{array}$ & $7.6(8.0)$ \\
\hline $\begin{array}{l}\text { Long half-life BDZs except for z-drugs (mg), } \\
\text { mean (SD) }\end{array}$ & $2.4(6.9)$ \\
\hline DZP equivalent dose of z-drugs $(\mathrm{mg})$, mean (SD) & $0.8(2.0)$ \\
\hline
\end{tabular}

Abbreviations: BDZ, benzodiazepine; CGI-S, Clinical Global Impressions-Severity of Illness scale; DZP, diazepam. regression analysis with a forward selection method. Regarding type A discrepancies, female sex and responses to Q9, Q12 and Q16 by psychiatrists approached statistical significance. Regarding type B discrepancies, responses to Q6 for patients, Q17 for patients and Q9 for psychiatrists approached statistical significance.

\section{Discussion}

\section{Main Findings and Highlights}

To the best of our knowledge, this is the first study to investigate differences in attitudes towards BDZ treatment between patients and psychiatrists. Of the patients in our survey, $13 \%$ perceived a reduction in the dose of BDZ to be more difficult than their referring psychiatrists did (Type A discrepancy), while $25 \%$ perceived it to be less difficult (Type B discrepancy). Agreement with Q9 (patient's desire), Q12 (short-term prescription), Q16 (rapport) for psychiatrists, and female sex of the patients were significantly associated with type A discrepancies. Furthermore, agreement with Q6 (necessity of psychotherapy plus BDZs), agreement with Q17 (dose increase) for patients and agreement with Q9 (patient's desire) for psychiatrists were significantly associated with type B discrepancies.

To assess the predictors of Type A and B discrepancies between patients and psychiatrists, we categorized the predictors into two groups based on the more pessimistic option. The predictors of pessimistic attitudes among patients regarding BDZ dose reductions were factors positively associated with type A discrepancies and negatively associated with type B discrepancies. However, the predictors of pessimistic attitudes among the referring psychiatrists were factors negatively associated with type A discrepancies and positively associated with type B discrepancies.

The female sex, patient agreement with Q6 (necessity of psychotherapy plus BDZs) and Q17 (dose increasing), and psychiatrist agreement with Q12 (short-term prescription) were categorized as predictors of pessimistic attitudes among patients toward BDZ dose reduction. The female sex is a known risk factor for the long-term prescription of $\mathrm{BDZs},{ }^{3,9-11}$ and pessimistic attitudes among patients regarding a dose reduction might lead to long-term use. A previous qualitative study indicated that discontinuation attempts by patients were common and not very successful, with rapid relapses. ${ }^{5}$ Psychiatrists might be unaware of the vicious cycle of long-term use, high-dose dependence, and 
Table 2 Patitent's Attitudes Toward BDZs

\begin{tabular}{|c|c|}
\hline Do you believe ... & Agree \\
\hline QI ... that dose reduction of BDZs is difficult? & $65.8 \%(102 / 155)$ \\
\hline Q2 ... that anxiolytics have efficacy for anxiety? & $88.4 \%(137 / 155)$ \\
\hline Q3 ... that hypnotics have efficacy for insomnia? & $87.1 \%(135 / 155)$ \\
\hline Q4 ... that anxiolytics have efficacy for pain? & $35.5 \%(55 / 168)$ \\
\hline Q5 ... that anxiolytics have efficacy for depressive mood? & $84.5 \%(131 / 155)$ \\
\hline Q6 ... that anxiolytics are necessary even with psychotherapy? & $81.9 \%(127 / 168)$ \\
\hline Q7 ... that psychotherapy takes long time? & $67.7 \%(105 / 155)$ \\
\hline Q8 for patients ... that psychotherapy is unsuitable for yourself? & $37.4 \%(58 / 155)$ \\
\hline Q8 for psychiatrists ... that psychotherapy is unsuitable for this patient? & \\
\hline Q9 for patients ... that the continued administration of BDZs should continue as long as you desire? & $89.0 \%(138 / 155)$ \\
\hline Q9 for psychiatrists ... that the continued administration of BDZs is justified because of the patient's desire? & \\
\hline $\begin{array}{l}\text { QI0 for patients ... that the continued administration of BDZs is justified as long as social functions (job/housekeeping/study) are maintained? } \\
\text { QI0 for psychiatrists ... that the continued administration of BDZs is justified because the patient's social functions are maintained? }\end{array}$ & $81.9 \%(127 / 155)$ \\
\hline QII for patients ... that the continued administration of BDZs is justified as long as adverse effects are not observed? & $84.5 \%(131 / 155)$ \\
\hline QII for psychiatrists ... that the continued administration of BDZs is justified because adverse effect have not been observed? & \\
\hline Q12 for patients ... that the continued administration of BDZs is justified for I month? & $55.5 \%(86 / 155)$ \\
\hline Q12 for psychiatrists ... that the continued administration of BDZs is justified for I month for this patient? & \\
\hline QI3 ... that information about addiction is important? & $80.0 \%(124 / 155)$ \\
\hline QI4 ... that BDZs increase falls? & $19.4 \%(30 / 155)$ \\
\hline QI5 ... that BDZs lead to daytime drowsiness? & $53.5 \%(83 / 155)$ \\
\hline Q16 ... that rapport breaks down without the administration of BDZs? & $34.8 \%(54 / 155)$ \\
\hline QI7 ... that the dose of BDZs has increased since the initial administration? & $25.8 \%(40 / 155)$ \\
\hline Q18 for patients Do you not want to take BDZs on principle? & $37.4 \%(58 / 155)$ \\
\hline Q18 for psychiatrists Do you not want to prescribe BDZs to this patient on & \\
\hline
\end{tabular}

Abbreviation: BDZs, benzodiazepines.

unsuccessful cessation attempts. This vicious cycle might lead to the associations between the discrepancies and the female sex and patient agreement with Q6 and Q17. Agreement with Q12 (short-term prescription) by the psychiatrists, which might reflect the psychiatrists' affirmative attitude toward the guidelines, was associated with pessimistic attitudes of patients toward BDZ dose reduction. Although we could not explain this association, the information gap between patients and psychiatrists might cause pessimistic attitudes among some patients.

In contrast, psychiatrist agreement with Q9 (patient's desire) and Q16 (rapport) could be considered predictors of pessimistic attitudes among referring psychiatrists. Psychiatrists' belief that patients desire BDZs (Q9) might differ from the patients' actual wishes, ${ }^{6}$ and such a belief might cause pessimistic attitudes among psychiatrists. In addition, patients might perceive that rapport with their psychiatrists relies on the provision of treatments other than simply BDZs, while psychiatrists might believe that rapport with their patients (Q16) only relies on their prescription of BDZs. This misconception might lead to discrepancies.

\section{Clinical and Demographic Characteristics as Risk Factors for the Perception of Difficulty in Reducing the Dose of BDZs}

In our results, the DZP equivalent dose of short half-life BDZs except for Z-drugs was associated with the patients' perception that a BDZ dose reduction would be difficult, while the duration of $\mathrm{BDZ}$ use was related to the referring psychiatrists' perception that a dose reduction would be difficult. There is modest evidence that short half-life BDZs are associated with a greater risk of dependence, ${ }^{12}$ and the pharmacological characteristics of BDZs might support our results. The duration of $\mathrm{BDZ}$ treatment is also known to be a risk factor for developing $\mathrm{BDZ}$ dependence. ${ }^{3}$

BDZ treatment is known to be a risk factor for traffic accidents, ${ }^{13}$ and long half-life BDZ anxiolytics are associated with a greater risk than short half-life BDZs. ${ }^{14}$ Furthermore, the concomitant use of alcohol and BDZs was found to increase the accident risk 7.7 times. ${ }^{15} \mathrm{We}$ could not completely explain why the patients' frequency of driving cars (every day) was related to the referring psychiatrists' perception of the difficulty 
Table 3 Psychiatrist's Attitudes Toward BDZs

\begin{tabular}{|c|c|c|}
\hline Do you believe ... & Agree & $\begin{array}{l}\text { Not } \\
\text { Applicable }\end{array}$ \\
\hline QI ... that dose reduction of BDZs is difficult? & $78.1 \%(121 / 155)$ & $0 \%(0 / 155)$ \\
\hline Q2 ... that anxiolytics have efficacy for anxiety? & $69.7 \%(108 / 155)$ & $14.2 \%(22 / 155)$ \\
\hline Q3 ... that hypnotics have efficacy for insomnia? & $75.5 \%(117 / 155)$ & $14.2 \%(22 / 155)$ \\
\hline Q4 ... that anxiolytics have efficacy for pain? & $12.9 \%(20 / 155)$ & $38.1 \%(59 / 155)$ \\
\hline Q5 ... that anxiolytics have efficacy for depressive mood? & $51.6 \%(80 / 155)$ & $18.1 \%(28 / 155)$ \\
\hline Q6 ... that anxiolytics are necessary even with psychotherapy? & $52.3 \%(81 / 155)$ & $14.8 \%(23 / 155)$ \\
\hline Q7 ... that psychotherapy takes long time? & $93.5 \%(145 / 155)$ & $2.6 \%(4 / 155)$ \\
\hline $\begin{array}{l}\text { Q8 for patients ... that psychotherapy is unsuitable for yourself? } \\
\text { Q8 for psychiatrists ... that psychotherapy is unsuitable for this patient? }\end{array}$ & $67.1 \%(104 / 155)$ & $0 \%(0 / 155)$ \\
\hline $\begin{array}{l}\text { Q9 for patients ... that the continued administration of BDZs should continue as long as you desire? } \\
\text { Q9 for psychiatrists ... that the continued administration of BDZs is justified because of the patient's desire? }\end{array}$ & $72.9 \%(113 / 155)$ & $0.6 \%(1 / 155)$ \\
\hline $\begin{array}{l}\text { Q10 for patients ... that the continued administration of BDZs is justified as long as social functions (job/housekeeping/study) are } \\
\text { maintained? }\end{array}$ & $74.8 \%(116 / 155)$ & $0.6 \%(1 / 155)$ \\
\hline Q10 for psychiatrists ... that the continued administration of BDZs is justified because the patient's social functions are maintained? & & \\
\hline $\begin{array}{l}\text { QII for patients ... that the continued administration of BDZs is justified as long as adverse effects are not observed? } \\
\text { QII for psychiatrists ... that the continued administration of BDZs is justified because adverse effect have not been observed? }\end{array}$ & $75.5 \%(117 / 155)$ & $0 \%(0 / 155)$ \\
\hline Q12 for patients ... that the continued administration of BDZs is justified for I month? & $39.4 \%(61 / 155)$ & $7.1 \%(11 / 155)$ \\
\hline Q12 for psychiatrists ... that the continued administration of BDZs is justified for I month for this patient? & & \\
\hline Q13 ... that information about addiction is important? & $98.1 \%(152 / 155)$ & $0 \%(0 / 155)$ \\
\hline QI4 ... that BDZs increase falls? & $5.2 \%(8 / 155)$ & $1.3 \%(2 / 155)$ \\
\hline Q15 ... that BDZs lead to daytime drowsiness? & $18.1 \%(28 / 155)$ & $0.6 \%(1 / 155)$ \\
\hline Q16 ... that rapport breaks down without the administration of BDZs? & $41.3 \%(64 / 155)$ & $0 \%(0 / 155)$ \\
\hline QI7 ... that the dose of BDZs has increased since the initial administration? & $35.5 \%(55 / 155)$ & $1.9 \%(3 / 155)$ \\
\hline Q18 for patients Do you not want to take BDZs on principle? & $65.8 \%(102 / 155)$ & $0 \%(0 / 155)$ \\
\hline Q18 for psychiatrists Do you not want to prescribe BDZs to this patient on principle? & & \\
\hline
\end{tabular}

Abbreviation: BDZs, benzodiazepines.

Table 4 Perception of Difficulty in Reducing BDZ Dose Among Patients and Psychiatrists Divided by Age and Gender

\begin{tabular}{|c|c|c|c|c|}
\hline & \multicolumn{4}{|c|}{ Number and \% } \\
\hline Age (Years) & $\leq 39$ & $\mathbf{4 0 - 5 4}$ & $\geq \mathbf{5 5}$ & Total \\
\hline Patients & & & & \\
Male sex & $8(47.1 \%)$ & $18(72.0 \%)$ & $7(58.3 \%)$ & $33(61.1 \%)$ \\
Female sex & $29(76.3 \%)$ & $15(68.2 \%)$ & $25(61.0 \%)$ & $69(68.3 \%)$ \\
\hline $\begin{array}{c}\text { Psychiatrists } \\
\text { Male sex }\end{array}$ & $14(82.4 \%)$ & $22(88.0 \%)$ & $10(83.3 \%)$ & $46(85.2 \%)$ \\
Female sex & $24(63.2 \%)$ & $16(72.7 \%)$ & $35(85.4 \%)$ & $75(74.3 \%)$ \\
\hline
\end{tabular}

involved in reducing the dose of BDZs. The relative affluence of the patients who owned their own car might have made the psychiatrists more optimistic. Despite the substantial risks associated with the concomitant use of BDZs and alcohol, ${ }^{16}$ a recent study from Japan reported that the prevalence of concomitant alcohol intake was $39.8 \% .{ }^{17}$ Psychiatrists might be pessimistic with regard to $\mathrm{BDZ}$ dose reductions in patients who habitually consume alcohol.

\section{Patients' or Psychiatrists' Attitudes as} Risk Factors for the Perception of Difficulty in Reducing the Dose of BDZs

Although $69.7 \%$ of the psychiatrists agreed with Q2 (efficacy of anxiolytics for anxiety), there is only low-quality evidence concerning the superiority of BDZs over a placebo for the short-term treatment of panic disorders. ${ }^{18}$ As first-line treatment for panic disorder, selective serotonin reuptake inhibitors are recommended in several guidelines. ${ }^{19-22}$ However, a study conducted in Japan showed a high prescription rate for BDZs, especially in the absence of concomitant antidepressant treatment, among patients with neurosis. ${ }^{23}$ In the case of patients with anxiety and major depression, there is no evidence of the efficacy of treatment with antidepressants plus BDZs with regard to the symptoms of anxiety. ${ }^{24}$ There might be misconceptions among psychiatrists regarding the efficacy of BDZ as a treatment for anxiety.

In the early phase (within 4 weeks) of treatment for major depression, there is moderate qualitative evidence that antidepressants plus BDZs reduce the severity of 
Table 5 Factors Associated with Difficulties with Reducing the Dose of BDZs According to Patients and Psychiatrists

\begin{tabular}{|c|c|c|c|c|c|c|}
\hline & B & Standard Error & Wald Value & P-value & Odds & Ratio \\
\hline \multicolumn{7}{|l|}{ Patients } \\
\hline DZP equivalent dose of short half-life BDZs except for $z$-drugs & 0.117 & 0.050 & 5.382 & 0.020 & 1.124 & $(1.018-1.241)$ \\
\hline Q6 for patients & 2.020 & 0.546 & 13.680 & $<0.001$ & 7.538 & $(2.585-21.987)$ \\
\hline Q16 for patients & 1.254 & 0.465 & 7.278 & 0.007 & 3.504 & $(1.409-8.715)$ \\
\hline QI7 for patients & 1.497 & 0.546 & 7.516 & 0.006 & 4.469 & $(1.532-13.031)$ \\
\hline \multicolumn{7}{|l|}{ Psychiatrists } \\
\hline Duration of BDZs use & 0.160 & 0.056 & 8.351 & 0.004 & 1.174 & $(1.053-1.309)$ \\
\hline \multicolumn{7}{|l|}{ Driving cars } \\
\hline \multicolumn{7}{|l|}{ Not at all (ref.) } \\
\hline Sometimes & -0.678 & 0.866 & 0.614 & 0.433 & 0.507 & $(0.093-2.769)$ \\
\hline Everyday & -2.475 & 0.863 & 8.234 & 0.004 & 0.084 & $(0.016-0.456)$ \\
\hline Habitual alcohol intake & 2.227 & 0.809 & 7.579 & 0.006 & 9.271 & $(1.899-45.252)$ \\
\hline Q2 for psychiatrists & 2.263 & 1.189 & 3.624 & 0.057 & 9.611 & $(0.935-98.754)$ \\
\hline Q5 for psychiatrists & -3.378 & 1.113 & 9.211 & 0.002 & 0.034 & $(0.004-0.302)$ \\
\hline Q9 for psychiatrists & 2.101 & 0.719 & 8.543 & 0.003 & 8.178 & $(1.998-33.466)$ \\
\hline Q16 for psychiatrists & 3.460 & 1.225 & 7.972 & 0.005 & 31.818 & $(2.88 I-351.406)$ \\
\hline QI7 for psychiatrists & 2.028 & 0.895 & 5.142 & 0.023 & 7.603 & $(1.317-43.892)$ \\
\hline Q18 for psychiatrists & 1.512 & 0.679 & 4.965 & 0.026 & 4.537 & $(1.200-17.158)$ \\
\hline
\end{tabular}

Abbreviations: BDZs, benzodiazepines; DZP, diazepam.

Table 6 Factors Associated with Patient-Psychiatrist Disagreement Regarding the Difficulties Involved in Reducing the Dose of BDZs

\begin{tabular}{|l|l|l|l|l|l|l|}
\hline & B & Standard Error & Wald Value & P-value & Odds & Ratio \\
\hline Type A discrepancy & & & & & & \\
$\quad$ Female sex & 2.220 & 0.832 & 7.113 & 0.008 & 9.205 & $(1.801-47.038)$ \\
Q9 for psychiatrists & -1.837 & 0.630 & 8.498 & 0.004 & 0.159 & $(0.046-0.548)$ \\
Q12 for psychiatrists & 1.520 & 0.635 & 5.724 & 0.017 & 4.572 & $(1.316-15.880)$ \\
Q16 for psychiatrists & -2.616 & 1.063 & 6.055 & 0.014 & 0.073 & $(0.009-0.587)$ \\
\hline Type B discrepancy & & & & & & \\
Q6 for patients & -2.390 & 0.564 & 17.971 & $<0.001$ & 0.092 & $(0.030-0.277)$ \\
Q17 for patients & -2.433 & 0.747 & 10.595 & 0.001 & 0.088 & $(0.020-0.380)$ \\
Q9 for psychiatrists & 1.290 & 0.611 & 4.461 & 0.035 & 3.634 & $(1.097-12.037)$ \\
\hline
\end{tabular}

Notes: Type A discrepancy: patients perceive more difficulties involved in reducing the dose of BDZs than their referring psychiatrists. Type B discrepancy: referring psychiatrists perceive more difficulties involved in reducing the dose of BDZs than patients.

Abbreviation: BDZs, benzodiazepines.

depressive symptoms. ${ }^{24}$ Several guidelines also recommended treatment with antidepressants plus BDZs within short-term (such as the first 4 weeks). ${ }^{25-27}$ In Japan, BDZs were prescribed to approximately $60 \%$ of patients with mood disorders. ${ }^{23}$ However, there is no evidence that treatment with antidepressants plus BDZs is effective for depressive symptoms over the course of 4 weeks, ${ }^{24}$ and long-term treatment with BDZs could lead to the development of dependence and accidents. ${ }^{12}$ Agreement with Q5 (efficacy of anxiolytics for depressive mood) on the part of psychiatrists was negatively associated with their perception of the difficulty of reducing the dose of BDZs (Q1). The psychiatrists' agreement with the guidelines might relate to their optimism with regard to the possibility of reducing the dose of BDZs.

There has been no evidence of a superior efficacy of psychotherapy plus BDZs compared with psychotherapy alone for patients with panic disorder. ${ }^{28}$ Agreement with Q6 (necessity of psychotherapy plus BDZs) on the part of patients was contrary to this lack of evidence, and this might lead these patients to believe that it would be difficult to reduce the dose of BDZs (Q1).

Agreement with Q9 (patient's desire) among psychiatrists was positively associated with their perception that it would be difficult to reduce the dose of BDZs (Q1), and 
previous studies support our results. A study regarding GPs' attitudes in Belgium reported that $47.2 \%$ of GPs thought patients would expect a prescription. ${ }^{7}$ Another study from Japan demonstrated that the main reason for difficulties with regard to hypnotic or anxiolytic dose reduction was that "patients were reluctant to reduce the dosages" ${ }^{29}$ In that study, more than half of the psychiatrists listed this reason as a barrier to reducing the doses of hypnotics or anxiolytics.

In each analysis, agreement with Q16 (rapport) was positively associated with the perception that it would be difficult to reduce the dose of BDZs (Q1). A previous study showed that patients were willing to stop treatment with BDZs; ${ }^{6}$ the psychiatric setting of our study in which participants might have more severe dependence could explain this difference. Japanese medical service payment data show that psychiatrist referrals were a significant risk factor for long-term hypnotic use compared with GP referrals. $^{3}$

In our study, agreement with Q17 (dose increase) on the part of both patients and psychiatrists was positively associated with their perception that it would be difficult to reduce the dose of BDZs (Q1). Japanese medical service payment data showed that the longer the duration of BDZ treatment was, the greater the subsequent increase in the dosage, ${ }^{3}$ which supported our results. Furthermore, agreement with Q18 (not wanting to prescribe BDZs on principle) on the part of psychiatrists was positively associated with their perception that it would be difficult to reduce the dose of BDZs (Q1). This might be caused by the psychiatrists' reluctance to renew prescriptions for BDZs written by other psychiatrists for patients with long-term BDZ use. A previous study showed that GPs felt that it was difficult to change colleagues' prescriptions of psychotropic drugs, ${ }^{30}$ which might also be true among psychiatrists.

\section{Perspective Regarding Reducing Long-Term BDZ Use and Overcoming Discrepancies in Attitudes}

Preventive psychoeducation at the time of the initial BDZ prescription is an important approach for preventing long-term BDZ use. However, for patients with longterm $\mathrm{BDZ}$ use, information regarding BDZ adverse effects should be carefully given to prevent abruptly ceasing and mitigate withdrawal. In addition, a patientcentered framework with prescriber-patient conversations is believed to reduce the dose of BDZs and prevent unintended abrupt cessation. ${ }^{31}$ Communication and interpersonal skills, such as warmth, trust, active listening and exploration of patient experiences, enhance prescriberpatient conversations. Patients must be involved in the decision-making process and should take time to prevent withdrawal. Practice guidelines that could be used for patient-centered care regarding reducing the use of psychotropic drugs need to be developed, and their efficacy needs to be investigated. Further studies investigating the promotion of patient-centered care to reduce BDZ use are needed.

\section{Strengths and Limitations}

There are several limitations of this study. First, our original questionnaire used in this study was not validated. Thus, the comparison and interpretation of our results should be conducted carefully. To investigate attitudes toward therapy, further studies with validated questionnaires, such as the 30-item Drug Attitude Inventory, are needed. ${ }^{32}$ Second, because our study was a cross-sectional study, this study could not elucidate the causal relationships between the predictors and the perception of the difficulty of BDZ dose reduction. Third, our participants were limited to those receiving long-term BDZ treatment. Thus, our results represent only the attitudes of patients with long-term BDZ use. Further studies investigating attitudes among patients receiving short-term BDZ treatment are needed. Fourth, several potential confounding factors, such as employment status, education status and previous BDZ cessation attempts indicated by the patients, were not assessed in our study. Other factors related to the sociodemographic conditions of the patients and the health organization were also not considered. Fifth, our sample was heterogeneous in psychiatric diagnoses. The treatment needs, adherence and perception can differ across different disorders. Thus, the interpretation of our results needs to consider this limitation. Sixth, we focused on psychiatrists and their patients who are not the main prescribers of BDZs. However, according to Japanese medical service payment data, patients referred to psychiatrists were at a significant risk of long-term hypnotic use compared with patients referred to GPs. ${ }^{3}$ Our study has the strength of considering the attitudes of long-term BDZ use patients. Furthermore, our study has the strength of considering the risk of dependence on BDZs, which, in contrast to other drug abuses, is often iatrogenic, without dose escalation and, therefore, often unnoticed and disregarded. ${ }^{31,33}$ 


\section{Conclusion}

Of the patients treated with BDZs for a year or longer, $65.8 \%$ reported that it would be difficult to reduce the dose of BDZ. Furthermore, 13\% of the patients perceived greater difficulty than their referring psychiatrists, while $25 \%$ of the patients perceived less difficulty. To overcome the differences in attitudes between patients and psychiatrists toward BDZ cessation, it is necessary to promote patient-centered care through patient psychoeducation and the provision of practice guidelines for decision-making process. Further studies on the promotion of patientcentered care to reduce BDZ use are needed.

\section{Data Sharing Statement}

The Ethics Committee of the Hirosaki University School of Medicine has set restrictions on data sharing because the data contain potentially identifying or sensitive patient information. Please contact the institutional review board of the ethics committee of the Hirosaki University School of Medicine for data requests. Upon request, the ethics committee will decide whether to share the data.

\section{Acknowledgments}

The authors would like to thank all of their coworkers for their helpful contributions to data collection and management.

\section{Funding}

The authors received no specific funding for this work.

\section{Disclosure}

Norio Yasui-Furukori reports personal fees from Mochida, Otsuka Pharmaceuticals, Dainippon-Sumitomo, and MSD, outside the submitted work. The remaining authors declare that they have no competing interests to report.

\section{References}

1. Soyka M. Treatment of Benzodiazepine Dependence. $N$ Engl J Med. 2017;376(12):1147-1157. doi:10.1056/NEJMra1611832

2. Takano A, Ono S, Yamana H, et al. Factors associated with long-term prescription of benzodiazepine: a retrospective cohort study using a health insurance database in Japan. BMJ Open. 2019;9(7):e029641. doi:10.1136/bmjopen-2019-029641

3. Enomoto M, Kitamura S, Tachimori H, Takeshima M, Mishima K. Long-term use of hypnotics: analysis of trends and risk factors. Gen Hosp Psychiatry. 2020;62:49-55. doi:10.1016/j.genhosppsych.20 19.11.008

4. Magnusdottir SD, Ribacke M. Patients' knowledge and attitudes regarding sleep and hypnotics. Scand J Prim Health Care. 1996;14 (2):106-110. doi:10.3109/02813439608997079
5. Liebrenz M, Gehring MT, Buadze A, Caflisch C. High-dose benzodiazepine dependence: a qualitative study of patients' perception on cessation and withdrawal. BMC Psychiatry. 2015;15:116. doi:10. 1186/s12888-015-0493-y

6. Williams F, Mahfouz C, Bonney A, et al. A circle of silence: the attitudes of patients older than 65 years of age to ceasing long-term sleeping tablets. Aust Fam Physician. 2016;45(7):506-511.

7. Anthierens S, Pasteels I, Habraken H, Steinberg P, Declercq T, Christiaens T. Barriers to nonpharmacologic treatments for stress, anxiety, and insomnia: family physicians' attitudes toward benzodiazepine prescribing. Can Fam Physician. 2010;56(11):e398-406.

8. Inada T, Inagaki A. Psychotropic dose equivalence in Japan. Psychiatry Clin Neurosci. 2015;69(8):440-447. doi:10.1111/pcn.12275

9. Blennow G, Romelsjö A, Leifman H, Leifman A, Karlsson G. Sedatives and hypnotics in Stockholm: social factors and kinds of use. Am J Public Health. 1994;84(2):242-246. doi:10.2105/AJPH.84.2.242

10. Neutel CI. The epidemiology of long-term benzodiazepine use. Int Rev Psychiatry. 2005;17(3):189-197. doi:10.1080/09540260500071863

11. Stowell KR, Chang CC, Bilt J, Stoehr GP, Ganguli M. Sustained benzodiazepine use in a community sample of older adults. $J \mathrm{Am}$ Geriatr Soc. 2008;56(12):2285-2291. doi:10.1111/j.1532-5415.20 08.02011.x

12. de Las Cuevas C, Sanz E, de la Fuente J. Benzodiazepines: more "behavioural" addiction than dependence. Psychopharmacology. 2003;167(3):297-303. doi:10.1007/s00213-002-1376-8

13. Elvik R. Risk of road accident associated with the use of drugs: a systematic review and meta-analysis of evidence from epidemiological studies. Accid Anal Prev. 2013;60:254-267. doi:10.1016/j. aap.2012.06.017

14. Barbone F, McMahon AD, Davey PG, et al. Association of road-traffic accidents with benzodiazepine use. Lancet. 1998;352 (9137):1331-1336. doi:10.1016/S0140-6736(98)04087-2

15. Dassanayake T, Michie P, Carter G, Jones A. Effects of benzodiazepines, antidepressants and opioids on driving: a systematic review and meta-analysis of epidemiological and experimental evidence. Drug Saf. 2011;34(2):125-156. doi:10.2165/11539050-000000000-00000

16. Tanaka E. Toxicological interactions between alcohol and benzodiazepines. J Toxicol Clin Toxicol. 2002;40(1):69-75. doi:10. 1081/CLT-120002887

17. Uchida T, Hirano J, Sakurai H, Suzuki T, Mimura M, Uchida H. Concomitant use of alcohol and benzodiazepine hypnotics in psychiatric outpatients: a cross-sectional survey. Int Clin Psychopharmacol. 2019;34(6):291-297. doi:10.1097/YIC.0000000 000000264

18. Breilmann J, Girlanda F, Guaiana G, et al. Benzodiazepines versus placebo for panic disorder in adults. Cochrane Database Syst Rev. 2019;3:CD010677. doi:10.1002/14651858.CD010677.pub2

19. American Psychiatric Association. American Psychiatric Association Practice Guideline for the Treatment of Panic Disorder. American Psychiatric Publishing; 2009. Available from: https://psychiatryon line.org/pb/assets/raw/sitewide/practice_guidelines/guidelines/panicdi sorder.pdf. Accessed 14 March 2021.

20. National Institute for Health and Care Excellence. Generalised Anxiety Disorder and Panic Disorder (With or Withou Agoraphobia) in Adults (CG113); 2011. Available from: https:// www.nice.org.uk/guidance/cg113. Accessed 14 March 2021.

21. Baldwin DS, Anderson IM, Nutt DJ, et al. Evidence-based pharmacological treatment of anxiety disorders, post-traumatic stress disorder and obsessive-compulsive disorder: a revision of the 2005 guidelines from the British Association for Psychopharmacology. J Psychopharmacol. 2014;28(5):403-439. doi:10.1177/02698811145 25674

22. Katzman MA, Bleau P, Blier $\mathrm{P}$, et al. Canadian clinical practice guidelines for the management of anxiety, posttraumatic stress and obsessive-compulsive disorders. BMC Psychiatry. 2014;14 Suppl 1 (Suppl1):S1. doi:10.1186/1471-244X-14-S1-S1 
23. Uchida H, Suzuki T, Mamo DC, et al. Benzodiazepine and antidepressant use in elderly patients with anxiety disorders: a survey of 796 outpatients in Japan. J Anxiety Disord. 2009;23(4):477-481. doi:10.1016/j.janxdis.2008.10.003

24. Ogawa Y, Takeshima N, Hayasaka Y, et al. Antidepressants plus benzodiazepines for adults with major depression. Cochrane Database Syst Rev. 2019;6:CD001026. doi:10.1002/14651858. CD001026.pub2

25. National Institute for Health and Care Excellence. Depression in adults with a chronic physical health problem: recognition and management (CG90); 2009. Available from: https://www.nice.org.uk/gui dance/CG90. Accessed 14 March 2021.

26. Cleare A, Pariante CM, Young AH, et al. Evidence-based guidelines for treating depressive disorders with antidepressants: a revision of the 2008 British Association for Psychopharmacology guidelines. J Psychopharmacol. 2015;29(5):459-525. doi:10.1177/0269881115 581093

27. Bauer M, Severus E, Möller HJ, Young AH. Pharmacological treatment of unipolar depressive disorders: summary of WFSBP guidelines. Int $J$ Psychiatry Clin Pract. 2017;21(3):166-176. doi:10.1080/13651501.2017.1306082

28. Watanabe N, Churchill R, Furukawa TA. Combined psychotherapy plus benzodiazepines for panic disorder. Cochrane Database Syst Rev. 2009;3(1):Cd005335.
29. Mishima K. Outline of the revision of medical service fees for preventing polypharmacy and long-term prescription of psychotropic drugs and its clinical background. Seishin Igaku. 2020;62 (4):365-375.

30. Svensson SA, Hedenrud TM, Wallerstedt SM. Attitudes and behaviour towards psychotropic drug prescribing in Swedish primary care: a questionnaire study. BMC Fam Pract. 2019;20(1):4. doi:10.1186/s12875-018-0885-4

31. Oldenhof E, Anderson-Wurf J, Hall K, Staiger PK. Beyond Prescriptions Monitoring Programs: the Importance of Having the Conversation about Benzodiazepine Use. J Clin Med. 2019;8(12):12. doi: $10.3390 /$ jcm8122143

32. Di Lorenzo R, Perrone D, Montorsi A, Balducci J, Rovesti S, Ferri P. Attitude Towards Drug Therapy in a Community Mental Health Center Evaluated by the Drug Attitude Inventory. Patient Prefer Adherence. 2020;14:995-1010. doi:10.2147/PPA.S251993

33. Willems IA, Gorgels WJ, Oude Voshaar RC, Mulder J, Lucassen PL. Tolerance to benzodiazepines among long-term users in primary care. Fam Pract. 2013;30(4):404-410. doi:10.1093/fampra/cmt010

\section{Publish your work in this journal}

Neuropsychiatric Disease and Treatment is an international, peerreviewed journal of clinical therapeutics and pharmacology focusing on concise rapid reporting of clinical or pre-clinical studies on a range of neuropsychiatric and neurological disorders. This journal is indexed on PubMed Central, the 'PsycINFO' database and CAS, and is the official journal of The International Neuropsychiatric Association (INA). The manuscript management system is completely online and includes a very quick and fair peer-review system, which is all easy to use. Visit http://www.dovepress.com/testimonials.php to read real quotes from published authors. 